Advanced Computing: An International Journal ( ACIJ ), Vol.2, No.2, March 2011

\title{
Computerized Shape Analysis Of Erythrocytes And Their Formed Aggregates In Patients Infected With P.Vivax Malaria
}

\author{
R.Renuka Devi ${ }^{\# 1}$, V.Rajagopal ${ }^{* 2}$, M.Senthil kumar ${ }^{\# 3}$, G.Magesh ${ }^{* 4}$ \\ \#1, \#3,*4, SITE Department, VIT University, \\ Vellore, Tamil nadu, India \\ ${ }^{1}$ renukadevi.r@vit.ac.in \\ ${ }^{3}$ senthilkumar.mohan@vit.ac.in \\ ${ }^{4}$ magesh.g@vit.ac.in \\ *2, Zonal Entomological Team, \\ Vellore, Tamil nadu, India \\ ${ }^{2}$ raja_vlr@yahoo.com
}

\begin{abstract}
Human plasmodial malaria is a severe infection of the erythrocytes with significant morbidity and mortality. The dimensional changes induced by P.vivax malaria parasites in erythrocytes in human blood are prominent and varies with the degree of parasitaemia. Aggregation of erythrocytes is a common finding in patients infected with malaria.

The changes in the shape of erythrocyte and its cytoplasm have been determined by shape descriptors and gray scale variation of the cytoplasm by microscopic imaging and image processing tools. The computerized shape analysis is carried out from the digital images obtained under microscope by shape descriptors based on projected area, perimeter and form factor,as measured by processing of images of erythrocytes in patients undergoing treatment for malaria. The changes induced in the cytoplasm by the malaria parasite are determined by the scanning of erythrocytes images along the horizontal diameter. The levels of aggregation of erythrocytes corresponding to the levels of infection, is measured and compared with normal samples. Growth of malaria parasite within the cytoplasm of the erythrocyte has also been measured. The measurable deviations in various other entities of the erythrocytes in a malaria patients using image processing techniques, has also been discussed.
\end{abstract}

Key words: Malaria, Plasmodium vivax, Shape analysis, Aggregates

\section{INTRODUCTION}

Malaria is a caused by parasites called plasmodia. Plasmodia parasites are transmitted between humans by the bite of an infected anopheles mosquito. It causes serious effects on human beings. Public health department and WHO took many steps to solve the drastic human losses. Malaria is one of the most serious parasitic infections of human. The accurate and timely diagnosis of malaria infection is essential to control and cure the disease. Despite the massive efforts put by the World Health Organization in the late 1950s and 1960s to eradicate malaria worldwide, the disease still remains a major tropical health problem, infecting over 200 million people worldwide. In India also malaria is a major health parasitic disease, where every year at least 2 million cases are reported. Malaria is one of the most serious public health problems in India and is prevalent in the whole of the country except areas above 1800 meters

DOI : $10.5121 /$ acij.2011.2207 
Advanced Computing: An International Journal ( ACIJ ), Vol.2, No.2, March 2011

above sea level. Recent reports have shown an increasing trend in the incidence of P.falciparum including the drug resistant strain and also spreading in areas earlier free from P.falciparum (Sharma et al., 1996).

Clinical features of malaria include fever rigors, anaemia, jaundice and splenomegaly that may occur in all human beings. In case of Plasmodium falciparum more sever complications may also ocuur. It is evident that no aspect of clinical picture of malaria is diagnostic, and all features including the complications of Plasmodium falciparum infection can be confused with other diseases.

In endemic areas, conventional blood smear is usually employed for diagnosis of the disease in hospitals and laboratories. The microscopic diagnosis is labour intensive and requires a high trained technician who can accurately examine 60-65 stained blood smears per day (Muqbil et al., 1998). During the recent years rapid serological methods for malarial diagnosis have been developed (Barker et al., 1992).

Considerable variations have been reported in the population of malaria parasites in respect of the morphology, infection pattern, susceptibility to mosquitos, cross-immunity, virulence, susceptibility to drugs, and antigen and enzyme variation.

Numbers of studies have been undertaken for the understanding of the interaction of malarial parasites with the host Red Blood Cells. (Miller et al., 2002) attempted to describe the mechanism of invasion of malarial merozoites into erthrocytes. The morphological changes induced by malarial parasited on the erythrocytes has been reportd (Aikawa et al., 1988). The influence of the Plasmodium vivax infection on the erythrocyte aggragation and deformability has also been reported (Sanjay Jajavanth et al., 2004)

It is well established that erythrocytes that have become infected with malarial parasites exhibit a number of fundamental changes in their plasma membrane properties as the parasites develop inside them. The morpho-functional changes in erythrocytes could further influence the erythrocyte shape and their aggregation. An attempt was made to employ shape analysis techniques to provide measurable parameter on the alterations induced by malarial parasites on the host red blood cells. An analysis based on these parameters can lead to a better understanding of some of the complications occurring in malaria infections and provide avenues for diagnosis and to track and cure malaria. Automation of detection of malaria parasites process will help in reducing the time taken for diagnosis and the chance for human errors.

\section{RELATED WORK}

Development of algorithm for detecting the severity of the disease. In computer aided medical diagnosis, Fuzzy logic and two soft computing tools can be used to identify the type and development stages of malarial parasites using thin blood smear image. Fuzzy c-mean clustering inorder can be used to classify the image into few image clusters. Histogram is used to know the information about the image directly. Then image segmentation is done using Threshold method. The second pixel found will be compared to the first one using Euclidean distance. The Euclidean value calculated will determine whether the second pixel will be grouped with the first pixel or it will be classified as a member of another cluster. Finally the number of malarial parasites was found. Screening of patients with various degrees of infection. Shape analysis of erythrocytes related to degree of parasitemia. Shape analysis before radical 
Advanced Computing: An International Journal ( ACIJ ), Vol.2, No.2, March 2011

therapy. Shape analysis during the course of the radical therapy. Shape analysis after radical therapy. Formation of hypothesis interpretation and discussion.

\section{MATERIAL AND METHODS}

The positive blood films on microslides were obtained from the malaria clinic at the Zonal Entomological Team at Vellore. Initially, the parasite density was determined on thick blood films by the conventional (+) system under the National Vector Borne Diseases Control Programme. Low, medium and high parasitemia blood samples were thus determined and stained using Leishman stain as per guidelines of the National Vector Borne Diseases Control Programme. Likewise blood films of normal individuals were taken for reference. Detection of malaria parasites in stained blood smears is critical for treatment of the disease (Makkapati, V.V. Rao, R.M.,2009).

The erythrocyte images were obtained by Leica DM2000 microscope at a magnification of 100x and simultaneously digitized by digital camera attached. The images were stored in the computer with Intel Pentium Core2Duo Processor at $1.50 \mathrm{GHz}$.

The entire shape analysis was carried out by employing the GUI using MATLAB-R2007b software supported with Windows 2000/XP operating system.

The steps involved in the algorithms for various measurements were determined and pretested to suit the study. Images are displayed on the monitor using the imread functions of the software. The RGB images are transformed to gray scale images. The images so obtained are filtered to avoid interferences in the background and to obtain the binary images. Edge enhancements were derived by employing the Sobel operators in the software. Contour extractions of the images by successive deletion of the outer most layer of the images was obtained for final measurements. The area and perimeter were determined based on the numbers of pixels. Based on the perimeter and area the Form Factor $(\mathrm{FF})=\mathrm{P} 2 / 4$. IA , was calculated. The FF was employed for determining the variation of cytoplasm gray level, aggregates analysis and erthrocyte shape analysis in the Low ,Medium and High pasitaemia patients before and after treatment for malaria. (Makkapati, V.V. Rao, R.M.,2009). Two binary images one with regions representing single cells and the second one containing red blood cells separated by the watershed technique. After identifying single cells and cell compounds, overlapping cells were separated using watershed transformation. And finally the RBC contours were calculated and output datas were generated (Dr. Ing. Jan Kybic, Prague, 2009). In analysis of infected cell using morphological operators, Segmentation was the important requirement of the system.(F.Boray Tek, Andrew G.Dempster, Izzet Kale, Westminster University, UK, 3 August,2009).

\section{RESULTS}

$\underline{\text { Shape analysis and description }}$

Image Output: Normal Erythrocyte 
Advanced Computing: An International Journal ( ACIJ ), Vol.2, No.2, March 2011
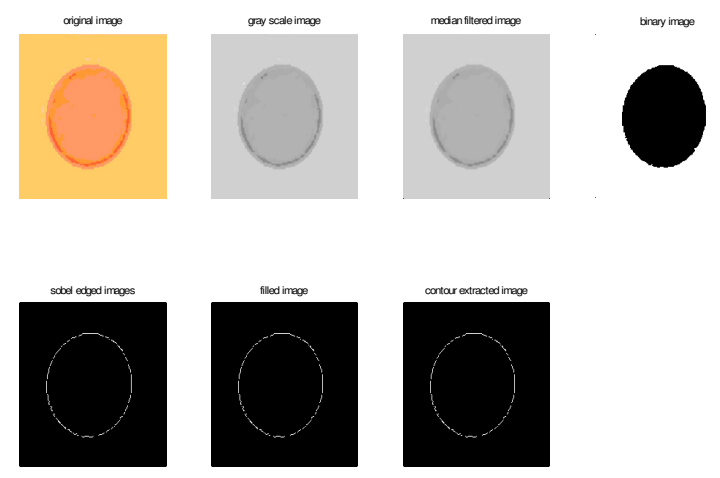

Fig1.Processed images of normal erythrocytes

Image Output: Medium Parasitemia

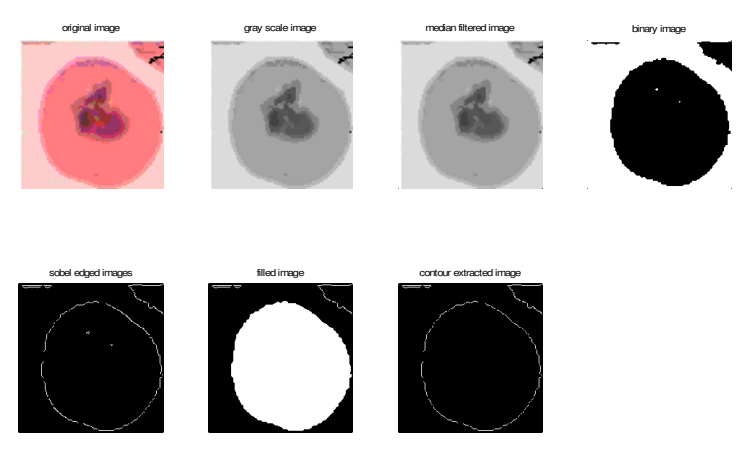

Fig2. Processed images of Medium parasitemia

\section{Image Output: Low Parasitemia}
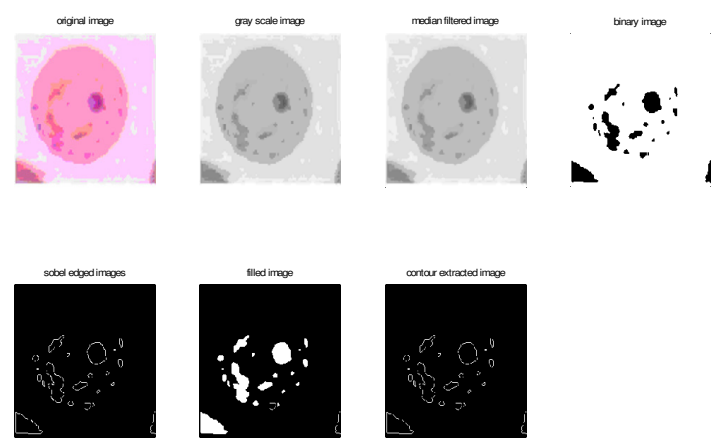

Fig3. Processed images of Low Parasitemia 
Advanced Computing: An International Journal ( ACIJ ), Vol.2, No.2, March 2011

Image Output: High Parasitemia
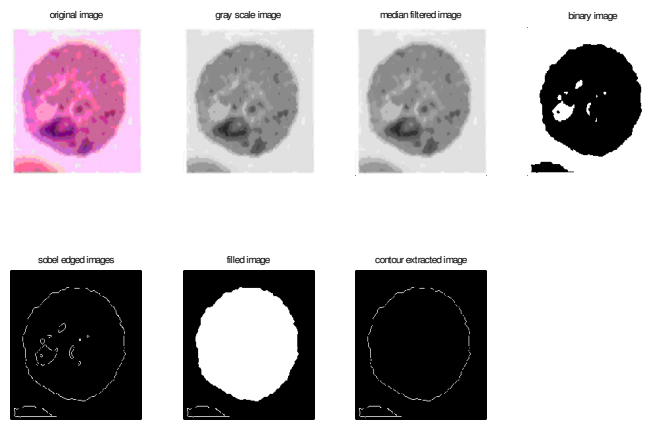

Fig4.Processed images of High parasitemia

\section{Variation of cytoplasm gray level}

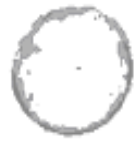

À

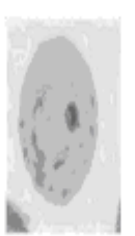

B

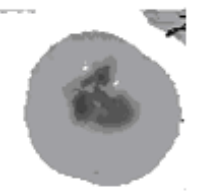

!.

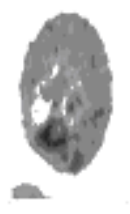

[

Figure5. Example of subtracted background cell images of (A) normal, and malaria samples of (B) low parasitemia, (C) medium parasitemia, (D) and high parasitemia

\begin{tabular}{|c|c|c|c|}
\hline $\begin{array}{c}\text { SAMPLES } \\
(\mathrm{n}=10)\end{array}$ & $\begin{array}{c}\text { AREA } \\
\left(\mu^{2}\right)\end{array}$ & $\begin{array}{c}\text { PERIMET } \\
\text { ER }(\mu \mathrm{m})\end{array}$ & $\begin{array}{c}\text { FF } \\
\left(\mathrm{P}^{2} / 4 p i A\right)\end{array}$ \\
\hline Normal & $\begin{array}{c}55.47 \pm 3.2 \\
\#\end{array}$ & $25.4 \pm 1.71$ & $0.97 \pm 0.11$ \\
\hline $\begin{array}{c}\text { Low } \\
\text { parasitemia }\end{array}$ & $\begin{array}{c}62.79 \pm 3.8 \\
1 *\end{array}$ & $\begin{array}{c}31.39 \pm 1.47 \\
*\end{array}$ & $1.26 \pm 0.14 *$ \\
\hline $\begin{array}{c}\text { Medium } \\
\text { parasitemia }\end{array}$ & $\begin{array}{c}64.99 \pm 2.9 \\
8 *\end{array}$ & $\begin{array}{c}33.55 \pm 1.74 \\
*\end{array}$ & $\begin{array}{c}1.57 \pm 0.38 * \\
*\end{array}$ \\
\hline $\begin{array}{c}\text { High } \\
\text { parasitemia }\end{array}$ & $\begin{array}{c}4 * 36 \pm 3.1 \\
4 * 3\end{array}$ & $\begin{array}{c}36.17 \pm 1.75 \\
* *\end{array}$ & $\begin{array}{c}1.35 \pm 0.22 * \\
*\end{array}$ \\
\hline
\end{tabular}

\# Mean \pm SD, *p $<0.05, * * p<0.01$

Table1. Respective variation in shape parameters of Erythrocytes of normal and malaria patients of low, medium and high parasitemia. 


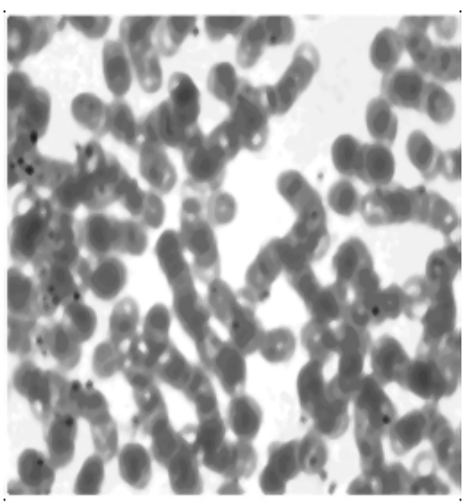

a

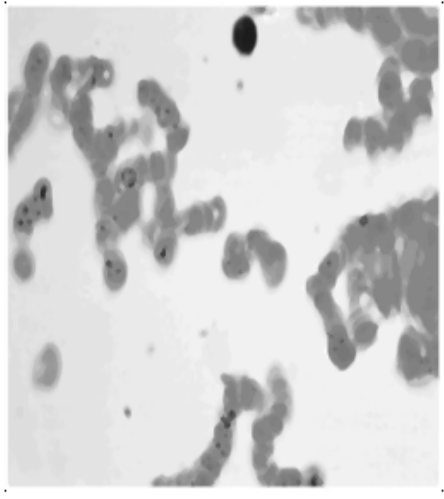

B

Figure6. Aggregates images of (A) normal, and (B) malaria subjects of low parasitemia.

\begin{tabular}{|l|c|}
\hline \multicolumn{1}{|c|}{ SAMPLES $(\mathrm{n}=10)$} & $\begin{array}{c}\text { AGGREGATES AREA } \\
\left(\text { pixels area in } \mu \mathrm{m}^{2} \text { ) }\right.\end{array}$ \\
\hline Normal & $8.22 \mathrm{E}+03 \pm 806.53 \#$ \\
\hline Low parasitemia & $4.61 \mathrm{E}+03 \pm 908.17^{*}$ \\
\hline
\end{tabular}

\#Mean+SD, *p $<0.05$

Table2. Comparison of the area occupied by aggregates of normal and malaria subjects.

\section{DISCUSSION AND CONCLUSION}

The clinical symptoms of malaria are a consequence of infection of human erythrocytes. The erythrocyte stages of malarial parasites induce morphological and functional changes in the infected erythrocytes. The host cell alterations are related to the capability of malarial parasites to alter the properties of the erythrocytes and its membranes. These include consumption and degradation of erythrocytic proteins, appearance of knob like protrusions in the membrane, electron dense material, clefts and vesicles in the cytoplasm. . A number of studies have been done for the understanding of the interaction of malarial parasites with the host erythrocytes. These include the hypothesis on the mechanism of erythrocytes invasion by malarial merozoites (Miller et al., 2002), the morphological changes induced by malarial parasites (Aikawa et al., 1988), the influence of P.vivax malaria on erythrocyte aggregation and deformability( Sanjay Jajavanth et al., 2004). 
Advanced Computing: An International Journal ( ACIJ ), Vol.2, No.2, March 2011

For humans the size and shapes of erythrocytes is important indicators of well being. The most significant factors responsible for micro and macro circulatory disorders are enhanced red blood cells aggregation, increased plasma viscosity and lowered erythrocytic deformability (AP Singh et al.,) Since aggregation and shape of red cells are a major determinants of normal flow properties of blood in microcirculation, in malaria the studies on automated methods to access deformabilities, and increased erythrocyte aggregation are essential for clinical evaluation and could be used as a marker of malaria status. To reduce the severity of the disease such analysis shall prove to be valuable in assessing the prevention or adhesion with anti-adhesive therapeutics (Sanjay Jajavanth et al., 2004), ( Jayavanth et al.,2003) Based on the measurd values of these parameters, the disease severity could further be estimated by neural network analysis (Sanjay Jajavanth et al., 2004).

\section{REFERENCES}

- Miller LH, Baruch DI, Marsh K, Doumbo OK,; The pathogenic basis of malaria-Nature (2002), 415; 673-679.

- Aikawa, Morphological changes in erythrocytes induced by malaria parasites, Biol.Cell. 84(1988), 173-182.

- Sanjay Jajavanth, K.Jagadeesan and Megha Singh; Influence of P.vivax malaria on erythrocyte aggregation and deformability, Clinical Hemorheology and Microcirculation 31 (2004) 257266.

- $\quad$ A.P.Singh,S.K.Puri and C.E.Chitnis, Antibodies raised against receptor-binding domain of Plamodium Knowlesi. Duffy binding protein inhibit erythrocyte invasion,Mol.Biochem.Parasitol. 121 (2002), 21-31.

- S.Jayavanth and M.Singh, Artificial neural network analysis of malaria severity through aggregation and deformability parameters of erythrocytes, Clin. Hemorheol. Microcirc. 29 (2003), 457-468.

- S.Sharma, A Differential clone of Plasmodium falciparum shows homology to the yeast SEC65 Domains, (1996), 177-180.

- N.A.Muqbil,Comparative study of antigen detection(ELISA-Method) and microscopical examination for plasmodium falciparum,(1998),15-17.

- Barker Jr, A simple method to detect plasmodium falciparum directly from blood samples using the polymerase chain reaction.American journal of tropical medicine and hygiene 46:416-426.

- Makkapati, V.V. Rao, R.M., Segmentation of malaria parasites in peripheral blood smear images, (2009).

- Automatic Malaria Diagnosis through Microscopy Imaging By Vít Springl, Supervisor: Dr. Ing. Jan Kybic, Prague, (2009).

- Parasite detection and identification for automated thin blood film malaria diagnosis F.Boray Tek, Andrew G.Dempster, Izzet Kale, Westminster University, UK, 3 August, (2009).

- Li-hui Zou Jie Chen Juan Zhang Garcia, Malaria cell counting Diagnosis within Large Field of View, (2010). 